\title{
Clostridioides difficile Diarrhea: An Emerging Problem in a South Indian Tertiary Care Hospital
}

\author{
Rachana Kannambath ${ }^{1}$ Rakhi Biswas ${ }^{1}$ Jharna Mandal \\ Narayanan Parameswaran ${ }^{4}$ \\ ${ }^{1}$ Department of Microbiology, Jawaharlal Institute of Postgraduate \\ Medical Education and Research, Puducherry, India \\ ${ }^{2}$ Department of Medicine, Jawaharlal Institute of Postgraduate \\ Medical Education and Research, Puducherry, India \\ ${ }^{3}$ Department of Medical Oncology, Jawaharlal Institute of \\ Postgraduate Medical Education and Research, Puducherry, India \\ ${ }^{4}$ Department of Paediatrics, Jawaharlal Institute of Postgraduate \\ Medical Education and Research, Puducherry, India
}

\begin{abstract}
Address for correspondence Rakhi Biswas, MD, Department of Microbiology, Jawaharlal Institute of Postgraduate Medical Education and Research, Puducherry - 605006, India (e-mail: dr.rakhibiswas@gmail.com).
\end{abstract}

J Lab Physicians 2021;13:346-352.

\begin{abstract}
Keywords

- Clostridioides difficile infection

- hospital-acquired diarrhea

- toxin detection

Context Clostridioides difficile infection (CDI) is one of the most common infectious causes of hospital-acquired diarrhea. The actual burden of the disease is underestimated in India due to inadequate diagnostic methods and limited studies conducted. Aims The aim of this study was to determine the burden and risk factors of CDI among patients with hospital-acquired diarrhea.

Methods and Materials Stool specimen of patients (age $>1$ year) with hospital-acquired diarrhea were screened for glutamate dehydrogenase antigen and toxin using an enzyme immunoassay. If both antigen and toxin were present, it was reported as positive for toxigenic CDI. Samples positive for antigen and negative for toxin were further tested with Cepheid GeneXpert assay for detecting the toxin producing gene.

Results Of 75 patients (mean age $36.07 \pm 20.79,64 \%$ males), 14 (18.67\%) patients were positive for toxigenic Clostridioides difficile (C. difficile) and $3(4 \%)$ patients were nontoxigenic $C$. difficile. Addition of GeneXpert to the testing algorithm increased the yield of toxin detection in 5/14 patients who were negative by toxin assay. On analysis of risk factors, prolonged hospital stay was found to have significant association $(p$-value $=0.022)$. Patients with factors like intensive care unit stay, presence of diabetes mellitus as a comorbidity, and exposure to antibiotics like carbapenems and glycopeptides have been found to have a higher prevalence of CDI.

Conclusions The prevalence of CDI in our population was $18.67 \%$ and the major risk factor associated was prolonged hospital stay. The addition of GeneXpert for the detection of toxin gene increased the yield from 12 to $18.68 \%$.
\end{abstract}

\section{Introduction}

The occurrence of diarrhea among hospitalized patients is quite common. Of the infectious causes of diarrhea in hospitalized patients, Clostridioides difficile (C. difficile) serves as the leading etiological agent. ${ }^{1} C$. difficile infection (CDI) is defined by the presence of gastrointestinal symptoms with published online

July 9,2021
DOI https://doi.org/

$10.1055 / \mathrm{s}-0041-1731944$

ISSN $0974-2727$ (c) 2021. The Indian Association of Laboratory Physicians.

This is an open access article published by Thieme under the terms of the Creative Commons Attribution-NonDerivative-NonCommercial-License, permitting copying and reproduction so long as the original work is given appropriate credit. Contents may not be used for commercial purposes, or adapted, remixed, transformed or built upon. (https://creativecommons.org/licenses/by-nc-nd/4.0/).

Thieme Medical and Scientific Publishers Pvt. Ltd. A-12, 2nd Floor, Sector 2, Noida-201301 UP, India 
either a stool test positive for toxigenic $C$. difficile or a histopathological or colonoscopic evidence of pseudomembranous colitis. ${ }^{2}$ It presents with a wide spectrum of clinical manifestations, ranging from mild-to-moderate diarrhea to the more severe pseudomembranous colitis, toxic megacolon, and sepsis. ${ }^{2}$ It is also notorious for causing large outbreaks in health-care facilities and severe epidemics contributed by the hypervirulent strains. Any factor contributing to the disruption of gut microbiota is found to have an association with $\mathrm{CDI}$. Rise in CDI has been markedly noted following the widespread use of broad-spectrum antibiotics in clinical practice. $^{3}$ There are several diagnostic modalities available for the detection of CDI in the present era, which range from standard culture methods to the latest molecular methods. But none of them have proven to be efficacious or cost-effective as a standalone test. ${ }^{4}$ Thus, it is essential to build a testing algorithm that would meet diagnostic needs optimally.

Actual burden of CDI in India is underestimated, mainly due to inadequate diagnostic techniques. Studies conducted in India especially in South India related to CDI are limited and have shown a wide prevalence ranging from 7 to $30 \%$ among patients with diarrhea and 2 to $4 \%$ in asymptomatic patients. ${ }^{5}$ This study hence attempted to determine the burden of CDI among our hospitalized patients using a two-step testing algorithm, and also analyzed the various risk factors associated with CDI in our hospital

\section{Materials and Methods}

This cross-sectional analytical study was conducted in a tertiary care hospital in Southern part of India after obtaining approval from the Institute Ethics Committee for Human studies. Patients $>1$ year of age who presented with diarrhea any time after 48 hours of hospital admission till discharge were enrolled from the Departments of Medicine, Medical Oncology, and Pediatrics. Diarrhea was defined as passage of three or more unformed stools in 24 hours and persisting for at least 2 days. Patients using laxatives concurrently or used 2 days before diarrhea onset were excluded. All consecutive samples were taken till the sample size of 75 was reached. Sample size was calculated using open epi software version 3.01 taking into account the prevalence rate of $16 \%$ of CDI as observed by Vishwanath et al in $2013 .{ }^{6}$

Informed consent was obtained from each of the patients and stool samples were collected in clean leak proof containers. Information was also collected from the medical records wherever available, regarding the colonoscopic and histopathological examination findings of suspected patients. The stool specimens were tested following an algorithm as shown in - Fig. 1. It was initially screened using a rapid membrane enzyme immunoassay, C. DIFF QUIK CHEK COMPLETE (Tech laboratory, Inc. Abbott, Blacksburg, Virginia, United States) for simultaneous detection of both glutamate dehydrogenase $(\mathrm{GDH})$ antigen and toxin $\mathrm{A} / \mathrm{B}$ of $C$. difficile. Procedure was followed as per the kit protocol. If both GDH antigen and toxin were negative, the sample was reported as negative for $C$. difficile and, when both GDH antigen and toxin are positive, it was interpreted as positive for toxigenic

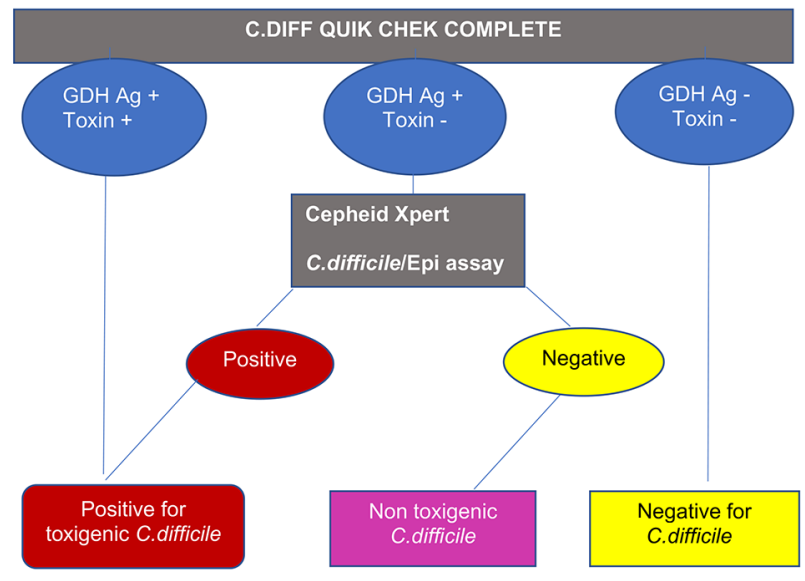

Fig. 1 A two-step testing algorithm for detecting the presence of both Clostridioides difficile (C. difficile) glutamate dehydrogenase $(\mathrm{GDH})$ antigen $(\mathrm{Ag})$ and toxin in stool samples.

C. difficile. All samples that were positive for GDH antigen and negative for toxin A/B were further tested with Cepheid Xpert C. difficile/Epi assay (Sunnyvale, California, United States), a cartridge-based real-time multiplex polymerase chain reaction (PCR) assay, for detecting "tcd B," a toxin producing gene present in $C$. difficile. Also, it was utilized for presumptive identification of the epidemic strain ribotype BI/NAP1/027 by detecting binary toxin gene sequences and the single base pair deletion at the nucleotide 117 in the tcd $C$ gene. The test was performed on the Cepheid GeneXpert Dx System according to the manufacturer's instruction. The results were recorded as: if the toxin gene was detected, the stool specimen was reported as positive for toxigenic $C$. difficile and if undetected, it was considered as nontoxigenic $C$. difficile. All the stool samples were also subjected to routine screening for parasitic ova and cyst, as well as, culture for detecting other bacterial pathogens.

\section{Statistical Analysis}

All categorical variables were expressed in terms of frequency and percentages. The association of outcome variable with these was done using the chi-squared test or Fisher's exact test. The continuous variables were expressed as mean with standard deviation or median with range based on normality and was compared with the outcome variable using independent Student's $t$-test or Mann-Whitney U test. All statistical analyses were carried at $5 \%$ level of significance and a " $p$-value" of $<0.05$ was considered statistically significant. To express the magnitude of association between the outcome variable and independent variables, prevalence ratio was used. Statistical analysis was performed using SPSS software version 19.

\section{Results}

\section{Demographic Profile, Clinical Features, and Comorbidities of the Patients with Hospital-Acquired Diarrhea}

A total of 75 patients were enrolled in the study as per the inclusion and exclusion criteria. Of the 75 patients, 43 
(57.3\%) were admitted in the Department of Medicine, 27 (36\%) in Medical Oncology, and $5(6.7 \%)$ in Pediatrics, while 37 (49.3\%) patients had intensive care unit (ICU) admission. Male to female ratio was $1.77: 1$. The mean age of the total population studied was $36.07 \pm 20.79$ years. Mean duration of hospital stay for the total population was 20 days, with a range of 5 to 93 days. Patients presented with a 4 days median duration of diarrhea with a range of 2 to 26 days. They also had a median frequency of 5 episodes of diarrhea per day with a range of 4 to 11 episodes. Out of 75 patients, 27 (36\%) had fever and 14 (18.7\%) had abdominal pain. None of them had any histopathological or colonoscopic evidence of pseudomembranous colitis. Also, 29 of the 75 patients (38.67\%) had malignancy as a comorbidity, with predominance of hematological malignancy (30.7\%). It was followed by diabetes mellitus (17.3\%) and chronic kidney disease (17.3\%). All the patients were on antibiotics-of the total 75 patients, 71 (94.7\%) patients were exposed to more than one antibiotic. Most common antibiotics in use were carbapenems (72\%) followed by $\beta$ lactam and $\beta$-lactamase inhibitors (57.3\%). The usage of third-generation cephalosporins and metronidazole were similar (42.7\%). Median duration of antibiotic exposure in the total population was 13 days with a range of 5 to 55 days. Of the total 75 patients, 66 (88\%) patients were on proton pump inhibitors (PPIs), 23 (30.7\%) on chemotherapeutic agents, 28 (37.3\%) on enteral tube feeding, and $15(20 \%)$ had history of previous hospitalization in the past 12 weeks.

\section{Laboratory Test Results}

Out of 75 samples, both GDH antigen and toxin were detected in 9 (12\%) samples, only GDH antigen was detected in $8(10.67 \%)$ samples by enzyme immunoassay (EIA). Out of these eight samples, toxin gene was detected in five samples by GeneXpert. Therefore, out of 75 samples, a total of $14(18.67 \%)$ samples were positive for toxigenic C. difficile, whereas 3 (4\%) samples were nontoxigenic $C$. difficile. None of the samples were found to be positive for the epidemic strain ribotype 027. Samples negative for GDH antigen and positive for the toxin were not found. In addition, stool wet mount and culture were also performed for all the samples. Hookworm egg and larva were detected in one sample each; three samples had cysts of Entamoeba histolytica/moshkovskii/dispar. In stool culture, Shigella species was isolated from one sample.

Table 1 Comparison between CDI-positive and CDI-negative population with respect to their demographic characters, clinical features, comorbidities, and laboratory results

\begin{tabular}{|c|c|c|c|c|}
\hline Parameter & $\begin{array}{l}\text { CDI positive } \\
(n=14)\end{array}$ & $\begin{array}{l}\text { CDI negative } \\
(n=61)\end{array}$ & $p$-Value & $\begin{array}{l}\text { Prevalence ratio } \\
(95 \% \mathrm{Cl})\end{array}$ \\
\hline \multicolumn{5}{|c|}{ Demographic characters and clinical features } \\
\hline Male gender & $11(78.6 \%)$ & $37(60.7 \%)$ & 0.208 & $2.06(0.62-6.75)$ \\
\hline ICU stay in days & $10(71.4 \%)$ & $27(44.3 \%)$ & 0.067 & $2.5(0.8-7.4)$ \\
\hline Age in years (mean with standard deviation) & $42.57 \pm 17.4$ & $34.57 \pm 21.3$ & 0.196 & - \\
\hline $\begin{array}{l}\text { Duration of hospitalization in days (median with } \\
\text { range) }\end{array}$ & $24.5(7-60)$ & $19(5-93)$ & 0.022 & - \\
\hline Number of days of diarrhea (median with range) & $3(2-26)$ & $4(2-15)$ & 0.397 & - \\
\hline Number of episodes of diarrhea (median with range) & $5(4-8)$ & $5(4-11)$ & 0.654 & - \\
\hline Presence of fever & $2(14.3 \%)$ & $25(41 \%)$ & 0.061 & $0.3(0.07-1.2)$ \\
\hline Presence of abdominal pain & $2(14.3 \%)$ & $12(19.7 \%)$ & 1 & $0.7(0.2-2.9)$ \\
\hline \multicolumn{5}{|c|}{ Drugs administration, presence of comorbidities, and other risk factors } \\
\hline Exposure to proton pump inhibitors & $13(92.9 \%)$ & $53(86.9 \%)$ & 1 & $1.77(0.26-11.9)$ \\
\hline Exposure to chemotherapeutic agents & $3(21.4 \%)$ & $20(32.8 \%)$ & 0.53 & $0.61(0.19-2)$ \\
\hline Malignancies & $3(21.4 \%)$ & $26(42.6 \%)$ & 0.14 & $0.43(0.13-1.42)$ \\
\hline Diabetes mellitus & $5(35.7 \%)$ & $8(13.1 \%)$ & 0.059 & $2.65(1.06-6.6)$ \\
\hline Chronic kidney disease & $3(21.4 \%)$ & $10(16.4 \%)$ & 0.69 & $1.3(0.42-4)$ \\
\hline Transplant & $1(7.1 \%)$ & $2(3.3 \%)$ & 0.47 & $1.85(0.35-9.85)$ \\
\hline Enteral tube feed & $6(42.9 \%)$ & $22(36.1 \%)$ & 0.64 & $1.25(0.48-3.25)$ \\
\hline Prior hospitalization & $3(21.4 \%)$ & $12(19.7 \%)$ & 1 & $1.09(0.34-3.24)$ \\
\hline \multicolumn{5}{|c|}{ Laboratory parameters } \\
\hline TLC (cubic mm)-median with range & $\begin{array}{l}11,345 \\
(1165-57,380)\end{array}$ & $\begin{array}{l}8,290 \\
(50-57,290)\end{array}$ & 0.14 & - \\
\hline Serum glucose $(\mathrm{mg} / \mathrm{dL})$-mean with SD & $98.21 \pm 38.4$ & $100.08 \pm 43.9$ & 0.89 & - \\
\hline Serum creatinine $(\mathrm{mg} / \mathrm{dL})$-median with range & $\begin{array}{l}0.98 \\
(0.47-11.39)\end{array}$ & $0.9(0.39-10.3)$ & 0.78 & \\
\hline Serum albumin(g/L)-median with range & $3(2-4)$ & $3(1-4)$ & 0.44 & - \\
\hline
\end{tabular}

Abbreviations: CDI, Clostridioides difficile infection; Cl, confidence interval; ICU, intensive care unit; SD, standard deviation; TLC, total lymphocyte count. 
All the positive findings in stool wet mount and culture were detected in samples that were negative for $C$. difficile.

\section{Characteristics of CDI-Positive Patients}

Out of 14 patients with CDI, 11 (78.6\%) patients were from medicine, 3 (21.4\%) patients were from medical oncology, while majority were males (78.6\%) and belonged to the age group > 18-45 years (64.3\%). Median duration of hospitalization was 24.5 days with a range of 7 to 60 days. Majority of them (10/14) were admitted in the ICU. Median duration of diarrhea was 3 days with a range of 2 to 26 days and the median frequency of diarrhea per day was 5 episodes with a range of 4 to 8 (-Table 1 ). Out of 14 patients, 2 (14.3\%) patients each had associated fever and abdominal pain, 13 (92.9\%) patients were on multiple antibiotics and PPIs, and $3(21.4 \%)$ patients were on chemotherapeutic agents (-Table 1). Majority of the CDI-positive patients were treated with carbapenems (85.7\%) followed by glycopeptides (57.1\%; - Table 2). Median duration of antibiotic exposure among patients with CDI was 15 days with a range of 5 to 45 days (-Table 2). Out of the 14 patients, 3 (21.4\%) patients had hematological malignancy, 5 (35.7\%) patients were diabetics, 3 (21.4) patients had chronic kidney disease, 1 patient had undergone bone marrow transplantation, 6 (42.9\%) patients were on enteral tube feeding, and $3(21.4 \%)$ patients had history of previous hospitalization ( $\mathbf{- T a b l e} \mathbf{1}$ ). A comparison has been made between CDI-positive and CDI-negative population with respect to their demographic characters, clinical features, antibiotic exposure, comorbidities, and laboratory results illustrated by - Table 1 and antibiotic exposure comparison between the two groups is depicted in - Table 2 . All the CDI-positive patients responded to treatment by metronidazole.

\section{Discussion}

From our study, the burden of CDI in our population was estimated at $18.67 \%$, which is similar to some of the studies conducted in India. A prevalence of $17 \%$ was observed in a study conducted by Ingle et al in a hospital in Mumbai. ${ }^{7}$ Similarly, a prevalence of $19.8 \%$ was obtained by Chakraborty et al in a study conducted on patients with antibiotic-associated diarrhea in Kolkata. ${ }^{8}$ Kumar and Uma in his study had observed a prevalence of $27 \%$ using combined EIA for GDH antigen and toxin. ${ }^{9}$ A study conducted by Segar et al in the same region as ours in 2017 had shown a prevalence of $4 \%$ that was very low compared with our study. ${ }^{10}$ This can be explained due to the difference in population studied and the difference in methodology adopted for testing. Our chosen study population were patients with hospital acquired diarrhea, whereas Segar et al had analyzed patients with acute diarrhea both from hospital and outpatients. They had also used combined EIA for the GDH antigen and toxin detection similar to ours for screening the stool samples. The addition of GeneXpert for the detection of toxin gene in our study increased the rate of toxigenic $C$. difficile detection from 12 to $18.68 \%$. Major

Table 2 Comparison between CDI-positive and CDI-negative population with respect to their antibiotic exposure

\begin{tabular}{|c|c|c|c|c|}
\hline Parameter & $\begin{array}{l}\text { CDI positive } \\
(n=14)\end{array}$ & $\begin{array}{l}\text { CDI negative } \\
(n=61)\end{array}$ & $p$-Value & $\begin{array}{l}\text { Prevalence ratio } \\
(95 \% \mathrm{CI})\end{array}$ \\
\hline $\begin{array}{l}\text { Exposure to multiple antimicrobial agents (> } 1 \\
\text { antibiotic) }\end{array}$ & $13(92.9 \%)$ & $58(95.1 \%)$ & 1 & $0.73(0.12-4.3)$ \\
\hline $\begin{array}{l}\text { Duration of exposure to antimicrobial agents in } \\
\text { days (median with range) }\end{array}$ & $15(5-45)$ & $12(5-55)$ & 0.33 & - \\
\hline \multicolumn{5}{|c|}{ Exposure to individual antibiotics } \\
\hline$\beta$-lactam $/ \beta$-lactamase inhibitors & $5(35.7 \%)$ & $38(62.3 \%)$ & 0.07 & $0.41(0.15-1.1)$ \\
\hline Third-generation cephalosporin & $5(35.7 \%)$ & $27(44.3 \%)$ & 0.56 & $0.74(0.27-2)$ \\
\hline Fourth-generation cephalosporin & $1(7.1 \%)$ & $2(3.3 \%)$ & 0.47 & $1.84(0.35-9.4)$ \\
\hline Carbapenems & $12(85.7 \%)$ & $42(68.9 \%)$ & 0.32 & $2.3(0.57-9.5)$ \\
\hline Aminoglycosides & $5(35.7 \%)$ & $25(41 \%)$ & 0.72 & $0.83(0.3-2.2)$ \\
\hline Fluoroquinolones & $2(14.3 \%)$ & $7(11.5 \%)$ & 0.67 & $1.22(0.32-4.6)$ \\
\hline Glycopeptides & $8(57.1 \%)$ & $21(34.4 \%)$ & 0.11 & $2.16(0.82-5.47)$ \\
\hline Macrolides & $1(7.1 \%)$ & $8(13.1 \%)$ & 0.42 & $0.56(0.08-3.8)$ \\
\hline Linezolid & $1(7.1 \%)$ & $12(19.7 \%)$ & 0.44 & $0.36(0.05-2.56)$ \\
\hline Clindamycin & $1(7.1 \%)$ & $4(6.6 \%)$ & 1 & $1.07(0.17-6.6)$ \\
\hline Colistin & $3(21.4)$ & $15(24.6 \%)$ & 1 & $0.86(0.27-2.75)$ \\
\hline Metronidazole & $5(35.7 \%)$ & 27 (44.3\%) & 0.56 & $0.74(0.27-2)$ \\
\hline Cotrimoxazole & $3(21.4 \%)$ & $8(13.1 \%)$ & 0.42 & $1.58(0.52-4.78)$ \\
\hline Amphotericin B & $1(7.1 \%)$ & $6(9.8 \%)$ & 1 & $0.74(0.11-8.49)$ \\
\hline Azoles & $3(21.4 \%)$ & 25 (41\%) & 0.17 & $0.45(0.14-1.5)$ \\
\hline Antivirals & $1(7.1 \%)$ & $8(13.1 \%)$ & 1 & $0.56(0.08-3.81)$ \\
\hline
\end{tabular}

Abbreviations: CDI, Clostridioides difficile infection; Cl, confidence interval; ICU, intensive care unit; SD, standard deviation; TLC, total lymphocyte count. 
disadvantage of using molecular methods for the detection of CDI is overdiagnosis of the cases. Even though they detect the toxin producing gene, the functionality of the gene or whether actually the organism is producing the toxin in vivo is unknown, since toxigenic strains are known to be colonizers of the gastrointestinal tract. Recently, an automated ultrasensitive toxin assay was approved by US Food and Drug Administration, which showed comparable sensitivity with the testing algorithm utilizing a GDH-and-toxin EIA and cell cytotoxicity neutralization assay..$^{11} \mathrm{~A}$ study was conducted for comparing the $C$. difficile toxin concentration in adults with symptomatic infection and asymptomatic carriage using the ultrasensitive quantitative immunoassay, which failed to yield any difference. ${ }^{12}$

The GeneXpert, however, in our study did not detect any hypervirulent ribotype 027 strain. But the possibility of its presence in our population cannot be ruled out because GeneXpert was not performed on samples that were already positive for toxin by EIA. Studies on the prevalence of strain types in India are rare. A study conducted by Vaishnavi et al, using both PCR for detection of various toxin genes and PCR ribotyping, found $9.2 \%$ of the strains to harbor the gene for one of the components of binary toxin either cdtA or cdtB. PCR ribotyping did not detect the presence of ribotype027. In the epidemic strain NAP1/BI/027, both the components of binary toxin gene are present. ${ }^{13}$

Male patients with CDI detected in our study were $78.6 \%$. But there was no statistically significant association between male gender and occurrence of CDI. The proportion of males among the total population analyzed was high (64\%) compared with females. This may be the reason for higher prevalence of CDI among males in this study. Various studies have shown different gender preponderance among CDI patients. Incidence of CDI has been found to be higher in females according to the data from Centers for Disease Control and Prevention's Emerging Infections Program surveillance conducted in 2011. ${ }^{14}$

CDI is mostly associated with advanced age ( $>65$ years) mainly due to presence of other comorbidities, increased exposure to medications, and more frequent hospitalization in this age group. ${ }^{15}$ Mean age of CDI in our population was $42.57 \pm 17.4$, compared with $34.57 \pm 21.3$ seen in non-CDI patients, which was not statistically significant. Prevalence of CDI among pediatric age group is also rising. ${ }^{15}$ In our study, $22.67 \%$ belonged to the age group greater than 1 and less than 18 years. Patients less than 1 year of age had been excluded from the study because of the high level of colonization of C. difficile in this group. Most common causative agents of diarrhea in this age group are the gastroenteric viruses. Due to these reasons, interpretation of a positive result of $C$. difficile becomes difficult. Proportion of CDI among the pediatric population (1-18 years) in our study was $5.9 \%$. In an Indian study conducted by Singh et al, a prevalence of $14 \%$ of CDI was observed in this age group. ${ }^{16}$

CDI was found 2.5 times more prevalent in patients who were admitted in ICU. This is in concordance with the study conducted by Ingle et $\mathrm{al}^{7}$ in Mumbai, Kim et al, ${ }^{17}$ and Cho et $\mathrm{al}^{18}$ in Korea. A retrospective case-control study of CDI among pediatric patients by Karaaslan et al showed a significant association between CDI occurrence and pediatric ICU stay. ${ }^{19}$ Duration of hospital stay in CDI patients prior to the onset of symptoms ranged from 7 to 60 days with a median of 24.5 days in our study. This was found to have a statistically significant association with the occurrence of CDI $(p$-value $=0.022)$. Similar results have been seen in a retrospective cohort study conducted in Australia by Selvey et al among patients with hematological malignancies, in which median number of days of admission was 29 days in CDI patients. ${ }^{20}$ Cho et al in his study had found a significant association between CDI and stay in long-term care facilities. ${ }^{18}$

All the patients in the CDI population as well as the non-CDI population were exposed to antibiotics in our study. Among the CDI-positive population, 92.9\% were on multiple antibiotics that were almost similar to non-CDI group, which had a multiple antibiotic exposure of $95.1 \%$. Prevalence of CDI was highest among the patients who were on carbapenems (prevalence ratio $=2.3$ ), followed by the glycopeptide group (prevalence ratio $=2.2$ ). Even though no statistically significant association was found between exposure to these antibiotics and occurrence of CDI, it is clinically significant. Carbapenems are broad-spectrum antibiotics that have action on anaerobes as well. Use of carbapenems could disrupt the normal anaerobic flora present in the intestine that could be the reason for higher prevalence among this population. In a study conducted by Lv et al, glycopeptides were found to have a protective effect against CDI contradictory to our study. ${ }^{21}$ Most of the studies have found significant association between the use of third-generation cephalosporins, clindamycin, and fluoroquinolones. ${ }^{3}$ In our study, 35.7\% patients were on third-generation cephalosporins, $7.1 \%$ were on clindamycin, and $14.3 \%$ were on fluoroquinolones. But there was no statistically significant association between exposure to any particular group of drug and occurrence of CDI. Association between use of PPIs and CDI has shown variations by different studies. ${ }^{22}$ Theoretically, acid suppression by PPI use can promote colonization as well as infection with C. difficile. In our study, 92.6\% of the CDI-positive population was exposed to PPI compared with $86.9 \%$ among non-CDI group, and was not statistically significant. Our results corroborate with the study of Tleyjeh et al, which demonstrated a weak association between PPI use and CDI in a systematic review and metanalysis. ${ }^{23}$ Patients exposed to chemotherapy are thought to be at more risk of CDI. In our study, $21.4 \%$ of the CDI patients were on chemotherapy, but did not have a statistically significant association. Similar observations were made in a case-control study conducted by Fueredes et al among hematooncology patients. ${ }^{24}$

Various underlying illnesses are considered as predisposing factor for CDI. In our study, CDI was 2.6 times more prevalent in patients with diabetes. Eliakim Raz et al conducted a retrospective case-control study in patients with diabetes and found that diabetics were at a higher risk of CDI compared with the general hospitalized patients (30 vs. 12\%). ${ }^{25}$ Malignancy especially hematological malignancies are more often associated with CDI. Ingle et al, ${ }^{7}$ Lall et $\mathrm{al}^{26}$ and Vaishnavi et $\mathrm{al}^{13}$ in their studies have found a 
significant association between presence of malignancy and occurrence of CDI. In our study, $21.4 \%$ of the CDI patients had hematological malignancies, but no statistically significant association was found. In a retrospective case-control study by Kim et al, the prevalence of $\mathrm{CDI}$ among patients with and without chronic kidney disease (CKD) was compared. It was found that patients with advanced stage of CKD and end-stage renal disease requiring dialysis were at two and three times more risk of acquiring CDI, respectively. ${ }^{17}$ In our study, $21.4 \%$ of CDI patients presented with chronic kidney disease compared with $16.4 \%$ among non-CDI patients. But this difference was also not found to be statistically significant. High risk of acquiring CDI is also witnessed among transplant patients. ${ }^{27}$ Selvey et al in their study conducted in patients with hematological malignancies found a significant association between CDI and autologous stem cell transplant. ${ }^{20}$ In our study, we had come across three patients who have undergone bone marrow transplantation, developing hospital acquired diarrhea, out of which only one patient was detected to be have CDI.

Enteral tube feeding and history of previous hospitalization are seen associated with higher incidence of CDI in many studies. Ingle et al in his study found a significant association between $\mathrm{CDI}$ and enteral tube feeding. ${ }^{28}$ Khanafer et al have conducted a study in French University, which revealed that patients with a history of previous hospitalization were at more risk of acquiring CDI. ${ }^{29}$ In our study, $42.9 \%$ of the patients with CDI were on enteral tube feeding compared with $36.1 \%$ in non-CDI cases. History of prior hospitalization within the past 12 weeks of illness was present in $21.4 \%$ of the CDI patients compared with $19.7 \%$ among non-CDI patients. However, no statistically significant difference was found in both the parameters.

Various laboratory parameters could guide in presumptive identification of CDI as well, as they are indicators of severity of disease. High leukocyte count, elevated serum creatinine, alanine aminotransferase level, and hypoalbuminemia are predictors of severe CDI. In our population, total leukocyte count was elevated in the CDI group (median of 11,345 with a range of 1,165-57,380 cubic $\mathrm{mm}$ ) compared with non-CDI group (median of 8,290 with a range of 50-57290 cubic mm). This did not account for a statistically significant difference. Laboratory parameters were mostly influenced by the underlying illnesses present in these patients in our study.

Small sample size was a major limitation in our study. Confining the study to only three departments in the hospital and absence of follow-up of the cases was also a disadvantage. Sensitizing clinicians to send samples for all cases of hospital-acquired diarrhea was also a challenge, as many of the cases were self-limiting and many subsided with initiation of empirical therapy with metronidazole.

\section{Conclusion}

From our study, it is evident that $\mathrm{CDI}$ is an emerging problem that cannot be neglected. It is under recognized in our country mainly due to the limitations of the existing diagnostic techniques. Adopting a suitable testing algorithm as well as having a good clinical correlation would further help in accurate diagnosis of CDI. Addition of molecular method improved the yield of toxin detection that was negative by toxin assay. Various factors have been associated with the occurrence of CDI. In our study, prolonged duration of hospitalization was found to be a statistically significant risk factor. Patients with factors like ICU stay, presence of diabetes mellitus as a comorbidity, exposure to antibiotics like carbapenems, and glycopeptides have been found to have a higher prevalence of CDI. A more extensive study, including a greater number of patients, preferably multicentric studies, would help to understand the predisposing factors of CDI better. Our study could be considered as a stepping stone toward that.

\section{Note}

This paper was previously presented at the 42nd Annual Conference of Indian Association of Medical Microbiologists (MICROCON 2018) held at Bengaluru, India from November 28 to December 2, 2018 (oral presentation).

\section{Consent to Participate}

Informed consent was obtained from all the adult participants included in the study; assent was obtained from children aged between 7 and 18 years. Consent was obtained from the legal guardian of all the participants below the age of 18 years.

\section{Ethics Statement}

This study was performed in line with the principles of the Declaration of Helsinki. Approval was granted by the Institute Ethics Committee of Jawaharlal Institute of Postgraduate Medical Education and Research, Puducherry (No. JIP/IEC/2016/10).

\section{Funding}

This study was funded by intramural research fund, Jawaharlal Institute of Postgraduate Medical Education and Research, Puducherry, India.

\section{Conflict of Interest}

The authors declare that they have no conflicts of interest.

\section{References}

1 Polage CR, Solnick JV, Cohen SH. Nosocomial diarrhea: evaluation and treatment of causes other than Clostridium difficile. Clin Infect Dis 2012;55(7):982-989

2 Cohen SH, Gerding DN, Johnson S, et al. Society for Healthcare Epidemiology of America; Infectious Diseases Society of America. Clinical practice guidelines for Clostridium difficile infection in adults: 2010 update by the Society for Healthcare Epidemiology of America (SHEA) and the Infectious Diseases Society of America (IDSA) Infect Control Hosp Epidemiol 2010;31(5):431-455

3 Eze P, Balsells E, Kyaw MH, Nair H. Risk factors for Clostridium difficile infections - an overview of the evidence base and challenges in data synthesis. J Glob Health 2017;7(1):010417

4 Burnham C-AD, Carroll KC. Diagnosis of Clostridium difficile infection: an ongoing conundrum for clinicians and for clinical laboratories. Clin Microbiol Rev 2013;26(3):604-630 
5 Verma P, Makharia GK. Clostridium difficile associated diarrhea: new rules for an old game. Trop Gastroenterol 2011;32(1):15-24

6 Vishwanath S, Singhal A, D'Souza A, Mukhopadhyay C, Varma M, Bairy I. Clostridium difficile infection at a tertiary care hospital in south India. J Assoc Physicians India 2013;61(11):804-806

7 Ingle $\mathrm{M}$, Deshmukh $\mathrm{A}$, Desai $\mathrm{D}$, et al. Prevalence and clinical course of Clostridium difficile infection in a tertiary-care hospital: a retrospective analysis. Indian J Gastroenterol 2011;30(2):89-93

8 Chakraborty A, Kar A, Roy S. Changing trends of Clostridium difficile associated diarrhoea (CDAD) in a tertiary care hospital in Kolkata, India. Intensive Care Med Exp 2015;3(Suppl 1) :A118

9 Kumar GV, Uma BM. Clostridium difficile: a neglected, but emerging pathogen in India. Arch Clin Microbiol 2015;6:2-6

10 Segar L, Easow JM, Srirangaraj S, Hanifah M, Joseph NM, Seetha KS. Prevalence of Clostridium difficile infection among the patients attending a tertiary care teaching hospital. Indian J Pathol Microbiol 2017;60(2):221-225

11 Landry ML, Topal JE, Estis J, Katzenbach P, Nolan N, Sandlund J. High agreement between an ultrasensitive Clostridioides difficile toxin assay and a C. difficile laboratory algorithm utilizing GDH-and-toxin enzyme immunoassays and cytotoxin testing. J Clin Microbiol 2020;58(2):e01629-e19

12 Pollock NR, Banz A, Chen X, et al. Comparison of Clostridioides difficile stool toxin concentrations in adults with symptomatic infection and asymptomatic carriage using an ultrasensitive quantitative immunoassay. Clin Infect Dis 2019;68(1):78-86

13 Vaishnavi C, Singh M, Mahmood S, Kochhar R. Prevalence and molecular types of Clostridium difficile isolates from faecal specimens of patients in a tertiary care centre. J Med Microbiol 2015;64(11):1297-1304

14 McDonald LC, Gerding DN, Johnson S, et al. Clinical practice guidelines for clostridium difficile infection in adults and children: 2017 update by the Infectious Diseases Society of America (IDSA) and Society for Healthcare Epidemiology of America (SHEA) Clin Infect Dis 2018;66(7):987-994

15 McFarland LV, Ozen M, Dinleyici EC, Goh S. Comparison of pediatric and adult antibiotic-associated diarrhea and Clostridium difficile infections. World J Gastroenterol 2016;22(11):3078-3104

16 Singh M, Vaishnavi C, Kochhar R, Mahmood S. Toxigenic Clostridium difficile isolates from clinically significant diarrhoea in patients from a tertiary care centre. Indian J Med Res 2017;145(6):840-846
17 Kim SC, Seo MY, Lee JY, et al. Advanced chronic kidney disease: a strong risk factor for Clostridium difficile infection. Korean J Intern Med (Korean Assoc Intern Med) 2016;31(1):125-133

18 Cho SM, Lee JJ, Yoon HJ. Clinical risk factors for Clostridium difficile-associated diseases. Braz J Infect Dis 2012;16(3):256-261

19 Karaaslan A, Soysal A, Yakut N, et al. Hospital acquired Clostridium difficile infection in pediatric wards: a retrospective case-control study. Springerplus 2016;5(1):1329

20 Selvey LA, Slimings C, Joske DJL, Riley TV. Clostridium difficile infections amongst patients with haematological malignancies: a data linkage study. PLoS One 2016;11(6):e0157839

21 Lv Z, Peng GL, Su JR. Factors associated with Clostridium difficile diarrhea in a hospital in Beijing, China. Braz J Med Biol Res 2014;47(12):1085-1090

22 Alzouby S, Baig K, Alrabiah F, Shibl A, Al-Nakhli D, Senok AC. Clostridioides difficile infection: Incidence and risk factors in a tertiary care facility in Riyadh, Saudi Arabia. J Infect Public Health 2020;13(7):1012-1017

23 Tleyjeh IM, Bin Abdulhak AA, Riaz M, et al. Association between proton pump inhibitor therapy and clostridium difficile infection: a contemporary systematic review and meta-analysis. PLoS One 2012;7(12):e50836

24 Fuereder T, Koni D, Gleiss A, et al. Risk factors for Clostridium difficile infection in hemato-oncological patients: a case control study in 144 patients. Sci Rep 2016;6:31498

25 Eliakim-Raz N, Fishman G, Yahav D, et al. Predicting Clostridium difficile infection in diabetic patients and the effect of metformin therapy: a retrospective, case-control study. Eur J Clin Microbiol Infect Dis 2015;34(6):1201-1205

26 Lall S, Nataraj G, Mehta P. Estimation of prevalence and risk factors for Clostridium difficile infection: a neglected pathogen in a tertiary care setting in India. Int. J. Med. Res. 2017;5:298-309

27 Alonso CD, Kamboj M. Clostridium difficile infection (CDI) in solid organ and hematopoietic stem cell transplant recipients. Curr Infect Dis Rep 2014;16(8):414

28 Ingle $\mathrm{M}$, Deshmukh $\mathrm{A}$, Desai $\mathrm{D}$, et al. Clostridium difficile as a cause of acute diarrhea: a prospective study in a tertiary care center. Indian J Gastroenterol 2013;32(3):179-183

29 Khanafer N, Vanhems P, Barbut F, et al. CDI01 Study groupTeams of. Factors associated with Clostridium difficile infection: a nested case-control study in a three year prospective cohort. Anaerobe 2017;44:117-123 